\title{
Some Examples of Weak Uninorms
}

\author{
Jiachao $\mathrm{Wu}^{1}$ and Yayan Yuan ${ }^{2}$ \\ ${ }^{1}$ Department of Mathematics, Shandong Normal University, Jinan 250014, China \\ ${ }^{2}$ College of Mathematics and Information Science, Henan Normal University, Xinxiang 453007, China
}

Correspondence should be addressed to Jiachao Wu; wujiachao1981@163.com

Received 24 May 2013; Revised 14 November 2013; Accepted 14 November 2013

Academic Editor: Natig M. Atakishiyev

Copyright (C) $2013 \mathrm{~J}$. Wu and Y. Yuan. This is an open access article distributed under the Creative Commons Attribution License, which permits unrestricted use, distribution, and reproduction in any medium, provided the original work is properly cited.

It is proved that, except for the uninorms and the nullnorms, there are no continuous weak uninorms who have no more than one nontrivial idempotent element. And some examples of discontinuous weak uninorms are shown. All of these examples are not $n$-uninorms, thus not uninorms or nullnorms.

\section{Introduction}

A mapping from $[0,1]^{2}$ to $[0,1]$ is called an AMC operator $[1,2]$, if it is associative, monotone nondecreasing in both variables, and commutative. The most famous AMC operator in fuzzy mathematics is the $t$-norms [3-5] and the $t$ conorms. In recent years, the weak forms of the $t$-norms interest the logisticians, such as the uninorms $[6,7]$, the nullnorms ( $t$-operators) $[8,9]$, the $n$-uninorms $[10,11]$, and the weak uninorms $[12,13]$.

It is easy to find that the weak uninorms are the most general class; that is, all the $t$-norms, the $t$-conorms, the $(n-)$ uninorms, and the nullnorms are weak uninorms. Conversely, it is not valid; that is, a weak uninorm could be none of the others [12].

As we all know, for a weak uninorm $R$, its idempotent elements are the points $x$ subject to $R(x, x)=x$. The elements 0 and 1 are the trivial idempotent elements of all the weak uninorms. All the common examples of the nontrivial weak uninorms are with infinite idempotents. Then, the following problem arises.

Problem 1 (see [14]). Is there a nontrivial weak uninorm with no more than one nontrivial idempotent elements?

This problem can be divided into two parts: continuous weak uninorms and discontinuous ones. In this paper, we will give answers to this problem separately.

The content will be arranged as follows: in Section 2, some basic definitions will be given, and it will be proved that there is no nontrivial continuous weak uninorms with none or one nontrivial idempotent element. In Section 3, some examples of weak uninorms with nontrivial idempotent elements are given. These examples give positive answers to the problem above. Section 4 also shows examples of weak uninorms, which have one or more idempotent elements. Section 5 gives a conclusion of this paper.

\section{Continuous Weak Uninorms}

Definition 2 (see $[3,4])$. An AMC operator $T$ is a $t$-norm, if $\forall x \in[0,1], T(x, 1)=x$.

An AMC operator $S$ is a $t$-conorm, if for any $x \in[0,1]$, $S(x, 0)=x$.

Definition 3 (see $[6,7]$ ). An AMC operator $U$ is said to be uninorm if there exists some element $e \in[0,1]$ called the neutral element such that for all $x \in[0,1], U(x, e)=x$.

Clearly, if $e=1$ then $U$ is a $t$-norm and if $e=0$ then $U$ is a $t$-conorm [7].

Definition 4. An AMC operator $V$ is called a nullnorm [8] ( $t$ operator [9]) if there exists an absorbing element $\lambda \in[0,1]$; that is, $V(x, \lambda)=\lambda$, such that for all $x \in[0, \lambda], V(x, 0)=x$ and for all $x \in[\lambda, 1], V(x, 1)=x$.

Obviously, if $\lambda=0$, then the nullnorm is a $t$-norm; and if $\lambda=1$, then it is a $t$-conorm. 
Definition 5 (see $[10,11]) .\left\{e_{1}, e_{2}, \ldots, e_{n}\right\}_{z_{1}, z_{2}, \ldots, z_{n-1}}$ is called an n-neutral element of an AMC operator $U_{n}$, if for $0=z_{0}<$ $z_{1}<\cdots<z_{n}=1$ and $e_{i} \in\left[z_{i-1}, z_{i}\right]$, we have $U_{n}\left(e_{i}, x\right)=x$, $\forall x \in\left[z_{i-1}, z_{i}\right]$.

An AMC operator is called an $n$-uninorm, if it has an $n$ neutral element $\left\{e_{1}, e_{2}, \ldots, e_{n}\right\}_{z_{1}, z_{2}, \ldots, z_{n-1}}$.

Obviously, each uninorm and nullnorm is an $n$-uninorm. And the converse is not valid. Examples could be found in [10].

Definition 6 (see $[12,13]$ ). An AMC operator $R$ is named a weak uninorm if for any $x \in[0,1]$, there exists some element $u_{x} \in[0,1]$ such that $R\left(x, u_{x}\right)=x$.

If $u_{x}$ in particular is a fixed value $e$ for all $x \in[0,1]$, then $e$ is called the neutral element of $R$. And in this case, $R$ comes to be uninorm [7]. If there is some element $\lambda \epsilon$ $[0,1]$, subject to for all $x \in[0, \lambda], u_{x}=0$, and for all $x \in$ $[\lambda, 1], u_{x}=1$, it is a nullnorm [8].

One can easily see that $n$-uninorms are weak uninorms. However, a weak uninorm may not be an $n$-uninorm and thus neither a nullnorm nor a uninorm. Examples are in $[12,13]$.

As a result, the problem in the introduction arises. And now, let us give an answer to it: there are no nontrivial continuous weak uninorms, but there exist discontinuous ones.

Theorem 7 (Theorem 7 in [15]). If $R$ is an AMC operator on $[0,1]$ that satisfies the following conditions:

(i) its idempotent elements are just 0 and 1;

(ii) there exists some element $x \in(0,1)$ with $R(x, x)>x$;

(iii) there exists some element $y \in(0,1)$ with $R(y, y)<y$;

then $R$ is not continuous, and $(d, d)$ is a discontinuous point, with $d$ the demarcation point.

From this theorem, we have the following theorem.

Theorem 8. Let $R$ be a continuous weak uninorm with no nontrivial idempotents. Then, we have the following results:

(1) if there exists some $x \in(0,1)$ subject to $R(x, x)<x$, then $R$ is a t-norm;

(2) if there exists some $x \in(0,1)$ subject to $R(x, x)>x$, then $R$ is a t-conorm.

Before the proof, let us show the following lemma firstly.

Lemma 9. Let $R$ be a continuous AMC operator with no idempotent elements except 0 and 1.

(1) If for all $x \in(0,1), R(x, x)<x$, then for any $x, y \in$ $(0,1)$, there exists some natural number $n$, subject to $y_{R}^{n}<x$.

(2) If for all $x \in(0,1), R(x, x)>x$, then for any $x, y \in$ $(0,1)$, there exists some natural number $n$, subject to $y_{R}^{n}>x$.
Proof. (1) Since for all $x \in(0,1), R(x, x)<x$, for any $n$,

$$
x_{R}^{n+1}=R\left(x_{R}^{2}, x_{R}^{n-1}\right) \leq R\left(x, x_{R}^{n-1}\right)=x_{R}^{n} .
$$

Thus, the limit $\lim _{n \rightarrow \infty} x_{R}^{n}$ exists, denoted by $\lim _{n \rightarrow \infty} x_{R}^{n}=$ $z$. From the continuity of $R$, we could know that

$$
z_{R}^{2}=\left(\lim _{n \rightarrow \infty} x_{R}^{n}\right)^{2}=\lim _{n \rightarrow \infty} x_{R}^{2 n}=z .
$$

This means that $z$ is an idempotent element of $R$. Because $z<$ $x, z=0$, that is, for any $x \in(0,1)$, the limit of $x_{R}^{n}$ is 0 . Thus, for any $x, y \in(0,1)$, there exists some natural number $n$, subject to $y_{R}^{n}<x$.

(2) It is similarly.

Now, let us show the proof of Theorem 8 .

Proof. Since a weak uninorm is an AMC operator. From Theorem 7, we could know that the squares of the elements in $(0,1)$ are either all strictly smaller than themselves or all strictly bigger than themselves; that is,

$$
\begin{aligned}
& \text { either } \forall x \in[0,1], \quad R(x, x)<x, \\
& \text { or } \forall x \in(0,1), \quad R(x, x)>x .
\end{aligned}
$$

(1) for all $x \in(0,1), R(x, x)<x$. Let's show that $R$ is a $t$-norm. From the definition of weak uninorms, for any $x \in(0,1)$, there is some $y \in[0,1]$, subject to $R(x, y)=x$. If $y \neq 1$, from Lemma 9 , there exists some $n$, subject to $y_{R}^{n}<x$. Thus, $x=R(x, y)=\cdots=$ $R\left(x, y_{R}^{n}\right) \leq R(x, x)<x$, contradiction. Therefore, $y=$ 1 ; that is, for any $x \in(0,1), R(x, 1)=x$. From the monotonicity of $R$, we could know that $R(0,1)=$ 0 and from the idempotence of 1 , we have $R(1,1)=1$. As a result,

$$
\forall x \in[0,1], \quad R(x, 1)=x,
$$

that is, $R$ is a $t$-norm.

(2) Similarly.

This theorem shows that there are no continuous weak uninorms with no nontrivial idempotents, except the $t$ norms and the $t$-conorms. For weak uninorms with just one nontrivial idempotent element, we have a similar result.

Theorem 10. There are no continuous weak uninorms with just one idempotent element $\lambda \in(0,1)$, except the uninorms and the nullnorms.

Proof. Suppose that $R$ is a continuous weak uninorm, with just one nontrivial idempotent element $\lambda$. Let's show that it is either a uninorm or a nullnorm.

Let $R^{\prime}(x, y)=R(\lambda x, \lambda y) / \lambda$, and $R^{\prime \prime}(x, y)=R((1-$ $\lambda) x+\lambda,(1-\lambda) y+\lambda) /(1-\lambda)$, for all $x, y \in[0,1]$. Obviously, $R^{\prime}$ and $R^{\prime \prime}$ are continuous AMC operators. Let's show that they are weak uninorms, that is, for each element $x, u_{x}$ exists. 
Since $\lambda, 0$, and 1 are idempotent elements and $R$ is continuous, from Theorem 2.8 in [14] (or Definition 3.1 in [9]), $R^{\prime}$ and $R^{\prime \prime}$ are continuous weak uninorms.

Obviously, they have no nontrivial idempotent elements. From Theorem 8, they are $t$-norms or $t$-conorms.

(1) If $R^{\prime}$ is a $t$-norm and $R^{\prime \prime}$ is a $t$-conorm, then $\lambda$ is the neutral element of $R$; that is, $R$ is a uninorm.

(2) If $R^{\prime}$ is a $t$-conorm and $R^{\prime \prime}$ is a $t$-norm, then $\lambda$ is an absorbing element $\lambda \in[0,1]$, and for all $x \in$ $[0, \lambda], V(x, 0)=x$ and for all $x \in[\lambda, 1], V(x, 1)=x$; that is, $R$ is a nullnorm.

(3) If both of them are $t$-norms, let us show that $R$ is a $t$-norm, a special uninorm. It just needs to show for all $x \in[0, \lambda]$,

$$
R(x, 1)=R(R(x, \lambda), 1)=R(x, R(\lambda, 1))=R(x, \lambda)=x \text {, }
$$

which could get that $R^{\prime}$ and $R^{\prime \prime}$ are $t$-norms.

(4) Similarly, if both of them are $t$-conorms, $R$ is also a $t$ conorm, a special uninorm.

\section{Weak Uninorms with No Nontrivial Idempotent Elements}

In this section, we will give some examples of weak uninorms, which have no nontrivial idempotent elements. And none of them is an $n$-uninorm; that is, all the examples in this section are nontrivial weak uninorms.

Example 11. The unit interval $[0,1]$ is divided into infinitely many sections as $\left(1-\left(1 / 2^{n-1}\right), 1-\left(1 / 2^{n}\right)\right]$, with $n=1,2, \ldots$. and 1 . Let $a_{n}=1-\left(1 / 2^{n-1}\right)$; define a mapping $R_{1}$ as follows:

$$
\begin{aligned}
& R_{1}(x, y) \\
& = \begin{cases}a_{n}+2^{n-1}\left(x-a_{n}\right)\left(y-a_{n}\right), & \text { if }(x, y) \in\left(a_{n}, a_{n+1}\right]^{2}, \\
1, & \text { if } x=1, \text { or } y=1, \\
\min \{x, y\}, & \text { otherwise. }\end{cases}
\end{aligned}
$$

Then, $R_{1}$ is a weak uninorm with no nontrivial idempotent elements; that is, its idempotent elements are just 0 and 1 . But it is not an $n$-uninorm and thus neither a uninorm nor a nullnorm.

Actually, for $(x, y) \in\left(a_{n}, a_{n+1}\right]^{2}, R_{1}(x, y)=a_{n}+\left((1 / 2) T_{P}\right.$ $\left.\left(2^{n}\left(x-a_{n}\right), 2^{n}\left(y-a_{n}\right)\right) / 2^{n}\right)$. For convenience, it is shortly rewritten as

$$
R_{1}(x, y)= \begin{cases}\frac{1}{2} T_{P}, & \text { if }(x, y) \in\left(a_{n}, a_{n+1}\right]^{2} \\ 1, & \text { if } x=1, \text { or } y=1, \\ \min \{x, y\}, & \text { otherwise. }\end{cases}
$$

In this formula, there is a symbol $(1 / 2) T_{P}$. It means an injection of the operator $(1 / 2) T_{P}$, that is, for all $(x, y) \in$ $\left(a_{n}, a_{n+1}\right]^{2}, R_{1}(x, y)=a_{n}+\left((1 / 2) T_{P}\left(2^{n}\left(x-a_{n}\right), 2^{n}\left(y-a_{n}\right)\right) / 2^{n}\right)$, instead of $R_{1}(x, y)=(1 / 2) T_{P}(x, y)$. Similar for the following examples.

Proof. Obviously, $R_{1}$ is monotone and commutative. Let's show it is associative.

For any $x, y, z \in[0,1]$, if one of them is 0 or 1 , then it is trivial.

If there is some $n$, subject to $x, y, z \in\left(a_{n}, a_{n+1}\right]$, from the associativity of $0.5 T_{P}, R_{1}$ is associative.

If there are some $m<n$, subject to $x, y \in\left(a_{n}, a_{n+1}\right]$ and $z \in\left(a_{m}, a_{m+1}\right]$, then we have $R_{1}(x, y)>a_{n} \geq a_{m+1} \geq$ $z, R_{1}(x, z)=z$ and $R_{1}(y, z)=z$. Therefore,

$$
R_{1}\left(R_{1}(x, y), z\right)=z=R_{1}(x, z)=R_{1}\left(x, R_{1}(y, z)\right) .
$$

If there are some $m<n$, subject to $x \in\left(a_{n}, a_{n+1}\right]$ and $y, z \in\left(a_{m}, a_{m+1}\right]$, then $R_{1}(x, y)=y, R_{1}(x, z)=z$ and $R_{1}(y, z)<a_{m+1} \leq a_{n} \leq x$. Thus, $R_{1}\left(x, R_{1}(y, z)\right)=R_{1}(y, z)$. Therefore,

$$
R_{1}\left(R_{1}(x, y), z\right)=R_{1}(y, z)=R_{1}\left(x, R_{1}(y, z)\right) .
$$

The last case is that $x \in\left(a_{n}, a_{\{n+1\}}\right], y \in\left(a_{m}, a_{\{m+1\}}\right]$ and $z \in\left(a_{l}, a_{\{l+1\}}\right]$, with different $l, m, n$. In this case, the result is always equal to the smallest element; that is, the associative law is valid. Now, the associativity of $R_{1}$ has already been proved.

Next, let us show that $R_{1}$ is a weak uninorm with no idempotent elements, except 0 and 1 .

For any $x \in(0,1)$, there is some $n$, subject to $x \in$ $\left(a_{n}, a_{n+1}\right]$. Let $y=a_{n+1}+\left(1 / 2^{n+3}\right)$, then $R_{1}(x, y)=x$. Together with $R_{1}(0,0)=0$ and $R_{1}(1,1)=1, R_{1}$ is a weak uninorm.

Since $(1 / 2) T_{P}(x, x)<x$, for all $x \in(0,1]$, we have $R_{1}(y, y)<y$, for all $y \in(0,1)$.

As a result, $R_{1}$ is a weak uninorm with idempotent elements no more than 0 and 1 .

It is obvious that it is not an $n$-uninorm, thus, neither a uninorm nor a nullnorm.

Note that it is not difficult to find that $\left([0,1),\left.R_{1}\right|_{[0,1)^{2}}\right)$, in which $\left.R_{1}\right|_{[0,1)^{2}}$ is the restriction of the weak uninorm $R_{1}$ on the square $[0,1)^{2}$, is the ordinal sum [16] of the semigroups $\left(\left(\left(1-\left(1 / 2^{n-1}\right), 1-\left(1 / 2^{n}\right)\right], T_{P}\right)\right)_{n \in N}$. But $R_{1}$ itself is not. Actually, similar to the proof of Example 11, we can obtain the following property.

Theorem 12. Let $R$ be an AMC operator on $[0,1]$ with $R(1,1)=1$. If $\left([0,1),\left.R\right|_{[0,1)^{2}}\right)$ is the ordinal sum of the semigroups $\left(\left(X_{\alpha}, T_{\alpha}\right)\right)_{\alpha \in A}$, in which $A$ is an infinite set, each $X_{\alpha}$ is in the form $(a, b]$, and each $T_{\alpha}$ is Archimedean, then $R$ is a weak uninorm with no idempotent elements, except 0 and 1.

Next, let us construct some more examples of weak uninorms. In these examples, if the ordinal sums are replaced as in this theorem, then they are still weak uninorms with no idempotent elements.

Example 13. The unit interval $[0,1]$ is divided into infinitely many sections as $\left[1 / 2^{n-1}, 1 / 2^{n}\right)$, with $n=1,2, \ldots$ and 0 . 
Then, the following defined $R_{2}$ is a weak uninorm with no non trivial idempotent elements:

$$
R_{2}(x, y)= \begin{cases}0.5+0.5 S_{P}, & \text { if }(x, y) \in\left[\frac{1}{2^{n}}, \frac{1}{2^{n-1}}\right)^{2} \\ 0, & \text { if } x=0, \text { or } y=0 \\ \max \{x, y\}, & \text { otherwise }\end{cases}
$$

that is, for $(x, y) \in\left[1 / 2^{n}, 1 / 2^{n-1}\right)^{2}, R_{2}(x, y)=\left(1 / 2^{n}\right)+(0.5+$ $\left.0.5 S_{P}\left(2^{n}\left(x-\left(1 / 2^{n}\right)\right), 2^{n}\left(y-\left(1 / 2^{n}\right)\right)\right) / 2^{n}\right)$.

$R_{2}$ is the dual of $R_{1}$. Thus, it is a weak uninorm.

Example 14. For some given $n_{0}$, define a mapping $R_{3}$ as follows:

$$
\begin{aligned}
& R_{3}(x, y) \\
& = \begin{cases}\min \{x, y\}, & \text { if } x=1, y \leq a_{n_{0}} \text { or } y=1, x \leq a_{n_{0}}, \\
R_{1}(x, y), & \text { otherwise, }\end{cases}
\end{aligned}
$$

in which $a_{n_{0}}=1-\left(1 / 2^{n_{0}}\right)$. Then, $R_{3}$ is a weak uninorm with idempotent elements 0 and 1 only.

Note that, in this example, if $a_{n_{0}} \neq 1-\left(1 / 2^{n_{0}}\right)$, the associativity will not be valid; that is, $R_{3}$ will no longer be a weak uninorm.

Example 15. In $R_{1}$, if $T_{P}$ is replaced by $T_{L}$, denoted as $R_{1}^{\prime}$, then it will no longer be a weak uninorm. Since the associativity is not valid,

$$
\begin{aligned}
& R_{1}^{\prime}\left(0.2, R_{1}^{\prime}(0.6,0.6)\right)=R_{1}^{\prime}(0.2,0.5)<0.2 \\
& R_{1}^{\prime}\left(R_{1}^{\prime}(0.2,0.6), 0.6\right)=R_{1}^{\prime}(0.2,0.6)=0.2
\end{aligned}
$$

This example shows that $T_{P}$ could not be replaced by any Archimedean $t$-norm.

Example 16. The following is a weak uninorm with only trivial idempotent elements:

$$
R_{4}(x, y)= \begin{cases}R_{1}, & \text { if }(x, y) \in[0,0.5)^{2}, \\ 1, & \text { if }(x, y) \in[0.5,1]^{2}, \\ \max \{x, y\}, & \text { otherwise. }\end{cases}
$$

The demarcation point of $R_{4}$ is 0.5 .

Example 17. Let $R_{5}$ be defined by

$$
\begin{aligned}
& R_{5}(x, y) \\
& = \begin{cases}R_{1}, & \text { if }(x, y) \in[0,0.5)^{2}, \\
\min \{x, y\}, & \text { if }(x, y) \in[0.5,1] \times[0,0.25] \\
1, & \cup[0,0.25] \times[0.5,1] \\
\max \{x, y\}, & \text { otherwise. }\end{cases}
\end{aligned}
$$

Then, $R_{5}$ is a weak uninorm with nontrivial idempotent elements. See Figure 1.

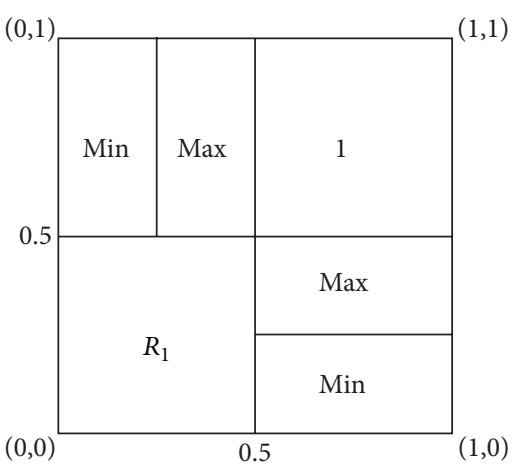

FIGURE 1: Graphical representation of $R_{5}$.

\section{Examples of Weak Uninorms with One or More Nontrivial Idempotent Elements}

Example 18. The following defined $R_{6}$ and $R_{7}$ are weak uninorms, with just one nontrivial idempotent element 0.5 :

$$
\begin{aligned}
& R_{6}(x, y)= \begin{cases}R_{1}, & \text { if }(x, y) \in[0,0.5)^{2}, \\
R_{1}, & \text { if }(x, y) \in[0.5,1)^{2}, \\
1, & \text { if } x=1, \text { or } y=1, \\
0.5, & \text { otherwise, }\end{cases} \\
& R_{7}(x, y)= \begin{cases}R_{1}, & \text { if }(x, y) \in[0,0.5)^{2}, \\
T_{L}, & \text { if }(x, y) \in(0.5,1]^{2}, \\
0.5, & \text { otherwise. }\end{cases}
\end{aligned}
$$

Example 19. Define a mapping $R_{8}$ by

$$
R_{8}(x, y)= \begin{cases}T_{P}, & \text { if }(x, y) \in[0,0.5)^{2}, \\ R_{1}, & \text { if }(x, y) \in(0.5,1]^{2} \\ \min \{x, y\}, & \text { otherwise. }\end{cases}
$$

Then, $R_{8}$ is a weak uninorm with idempotent elements $0,0.5$, and 1.

Examples 18 and 19 are constructed by $R_{1}$. The next example is not in this case.

Example 20. Define mappings $R_{9}$ and $R_{10}$ (see Figure 2 ) as follows:

$$
\begin{aligned}
& R_{9}(x, y) \\
& = \begin{cases}T_{P}, & \text { if }(x, y) \in[0.6,1]^{2}, \\
S_{P}, & \text { if }(x, y) \in[0.3,0.6]^{2} \\
\min \{x, y\}, & \text { if } x<0.3, y=1 ; \text { or } x=1, y<0.3, \\
0.6, & \text { if }(x, y) \in[0.6,1] \times[0.3,0.6] \\
& \quad \cup[0.3,0.6] \times[0.6,1], \\
0, & \text { otherwise, }\end{cases}
\end{aligned}
$$




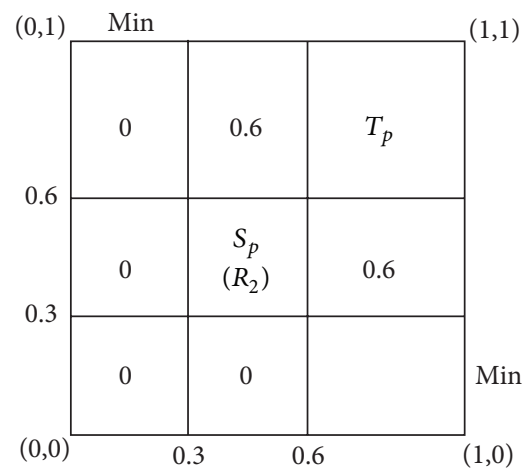

FIGURE 2: Graphical representation of $R_{9}\left(\right.$ or $\left.R_{10}\right)$.

$$
\begin{aligned}
& R_{10}(x, y) \\
& = \begin{cases}T_{P}, & \text { if }(x, y) \in[0.6,1]^{2}, \\
R_{2}, & \text { if }(x, y) \in(0.3,0.6]^{2} \\
\min \{x, y\}, & \text { if } x \leq 0.3, y=1 ; \text { or } x=1, y \leq 0.3, \\
0.6, & \text { if }(x, y) \in[0.6,1] \times[0.3,0.6] \\
0, & \quad \text { otherwise. }\end{cases}
\end{aligned}
$$

Then $R_{9}$ is a weak uninorm with two nontrivial idempotent elements 0.3 and $0.6 ; R_{10}$ is a weak uninorm with just one nontrivial idempotent element 0.6.

\section{Conclusion}

In this paper, it is proved that there are no nontrivial continuous weak uninorms with none or one idempotent element. Moreover, some nontrivial examples of weak uninorms are given. These examples are with no more than two nontrivial idempotent elements, which is a positive answer to the question in [14].

\section{Acknowledgment}

This project is supported by the Tianyuan special funds of the National Natural Science Foundation of China (Grant no. 11226265), Promotive research fund for excellent young and middle-aged scientists of Shandong Province (Grant no. 2012BSB01159), and Foundation of the Education Department Henan Province (no. 13A110552).

\section{References}

[1] M. Grabisch, J.-L. Marichal, R. Mesiar, and E. Pap, "Aggregation functions: means," Information Sciences, vol. 181, no. 1, pp. 1-22, 2011.

[2] M. Grabisch, J.-L. Marichal, R. Mesiar, and E. Pap, "Aggregation functions: construction methods, conjunctive, disjunctive and mixed classes," Information Sciences, vol. 181, no. 1, pp. 23-43, 2011.

[3] E. P. Klement, R. Mesiar, and E. Pap, Triangular Norms, Kluwer Academic, Dodrecht, The Netherlands, 2000.
[4] J. T. Starczewski, "Extended triangular norms," Information Sciences, vol. 179, no. 6, pp. 742-757, 2009.

[5] R. R. Yager, "Aggregation operators and fuzzy systems modeling," Fuzzy Sets and Systems, vol. 67, no. 2, pp. 129-145, 1994.

[6] J. C. Fodor, R. R. Yager, and A. Rybalov, "Structure of uninorms," International Journal of Uncertainty, Fuzziness and KnowledgeBased Systems, vol. 5, no. 4, pp. 411-427, 1997.

[7] R. R. Yager and A. Rybalov, "Uninorm aggregation operators," Fuzzy Sets and Systems, vol. 80, no. 1, pp. 111-120, 1996.

[8] T. Calvo, B. de Baets, and J. Fodor, "The functional equations of Frank and Alsina for uninorms and nullnorms," Fuzzy Sets and Systems, vol. 120, no. 3, pp. 385-394, 2001.

[9] M. Mas, G. Mayor, and J. Torrens, “T-operators," International Journal of Uncertainty, Fuzziness and Knowledge-Based Systems, vol. 7, no. 1, pp. 31-50, 1999.

[10] P. Akella, "Structure of $n$-uninorms," Fuzzy Sets and Systems, vol. 158, no. 15, pp. 1631-1651, 2007.

[11] P. Akella, " $C$-sets of $n$-uninorms," Fuzzy Sets and Systems, vol. 160, no. 1, pp. 1-21, 2009.

[12] Y.-M. Li and Z.-K. Shi, "Weak uninorm aggregation operators," Information Sciences, vol. 124, no. 1-4, pp. 317-323, 2000.

[13] Y.-M. Li and Z.-K. Shi, "Remarks on uninorm aggregation operators," Fuzzy Sets and Systems, vol. 114, no. 3, pp. 377-380, 2000.

[14] J. Wu and M. Luo, "Some properties of weak uninorms," Information Sciences, vol. 181, no. 18, pp. 3917-3924, 2011.

[15] J. Wu, "Some properties of AMC operators," Information Sciences. Revising.

[16] E. P. Klement, R. Mesiar, and E. Pap, "Triangular norms as ordinal sums of semigroups in the sense of A. H. Clifford," Semigroup Forum, vol. 65, no. 1, pp. 71-82, 2002. 


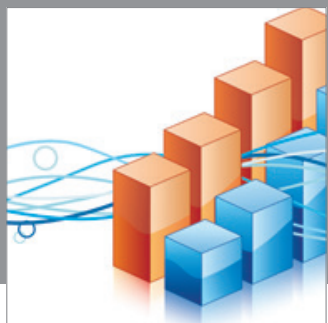

Advances in

Operations Research

mansans

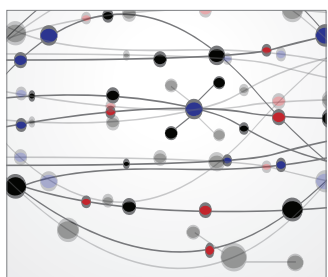

The Scientific World Journal
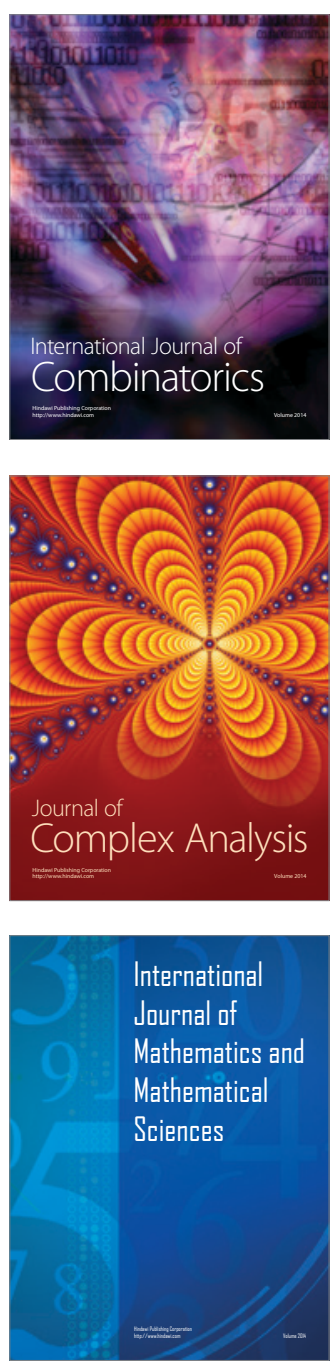
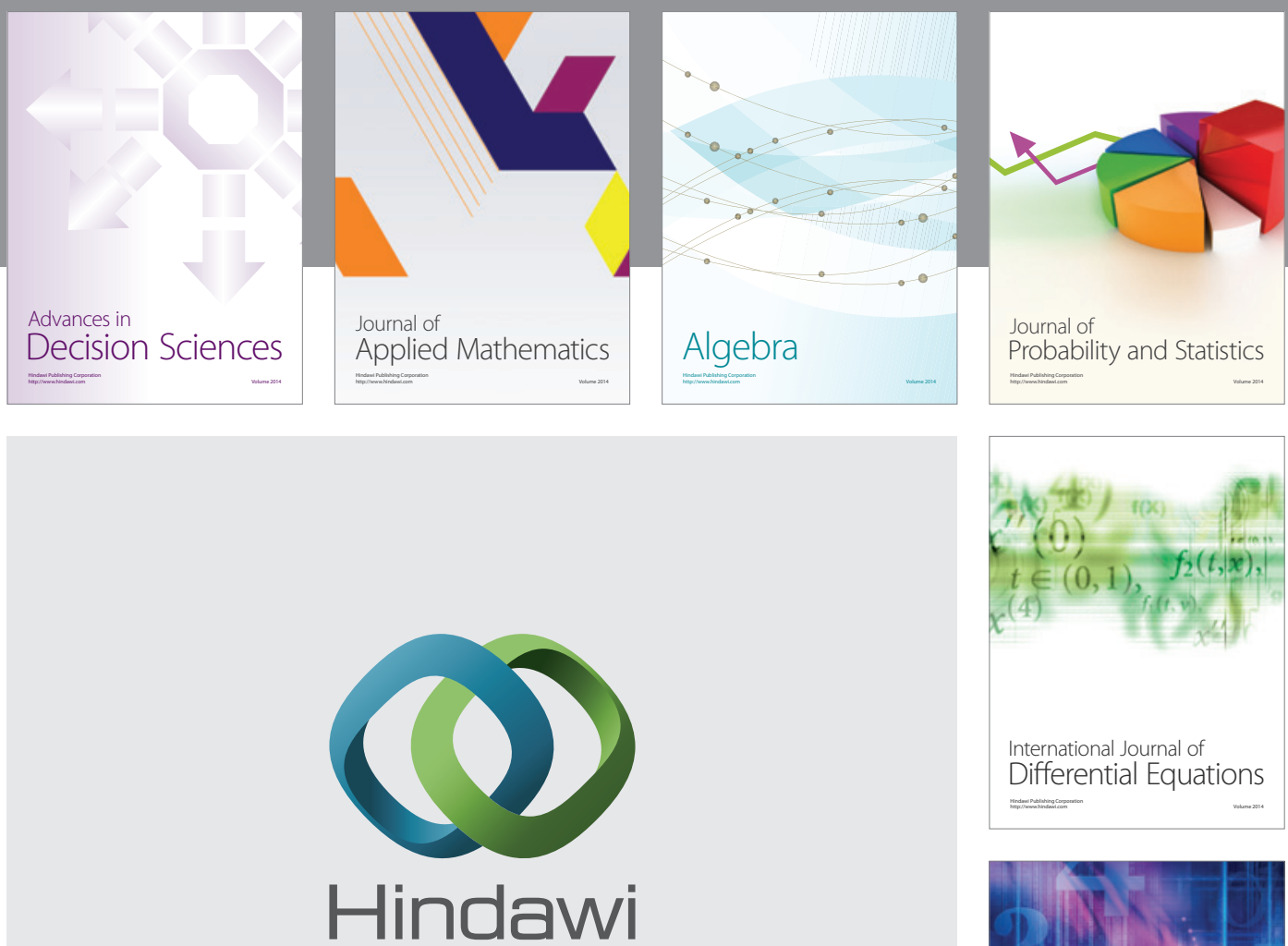

Submit your manuscripts at http://www.hindawi.com
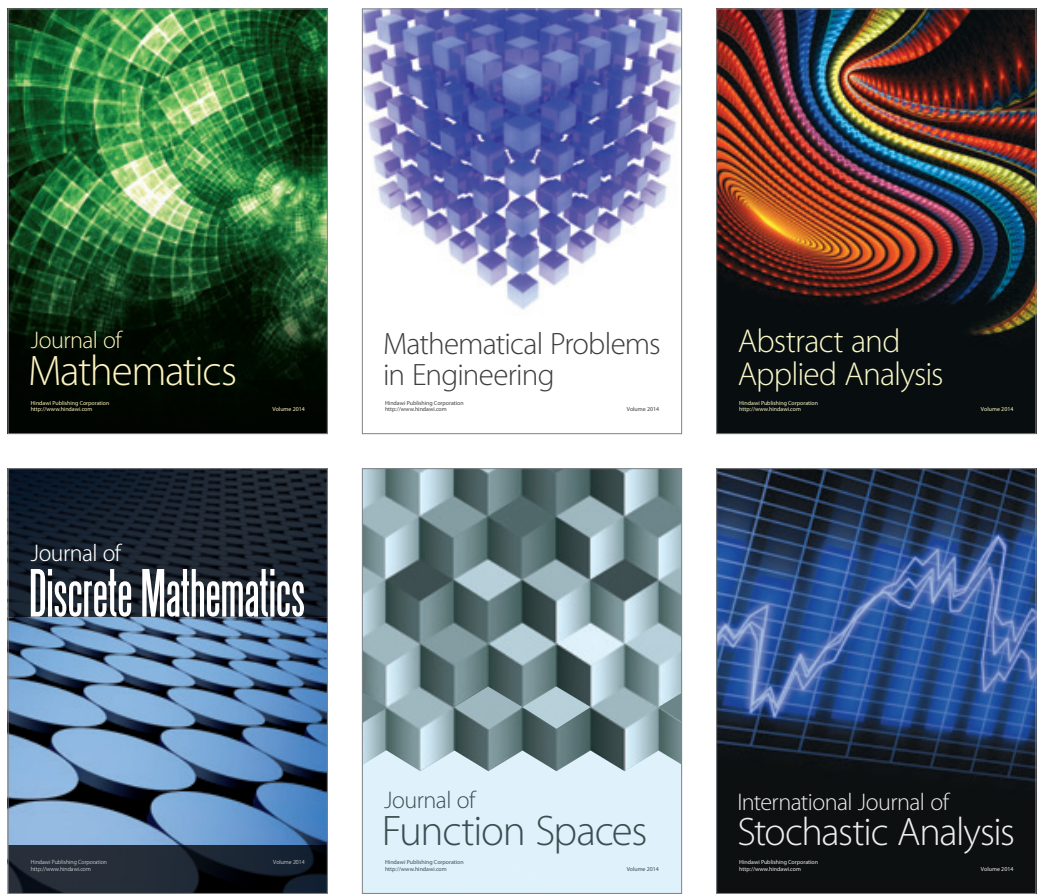

Journal of

Function Spaces

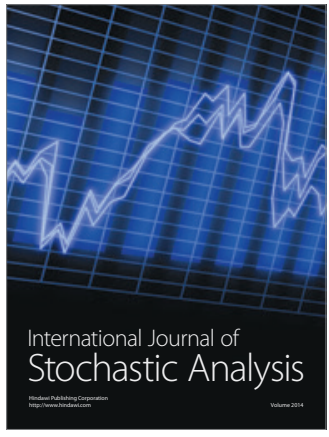

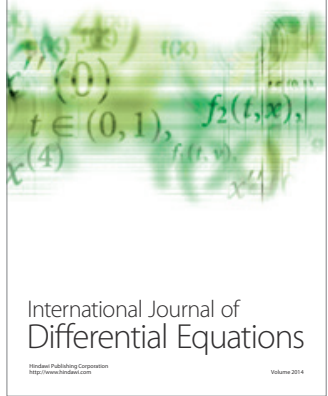
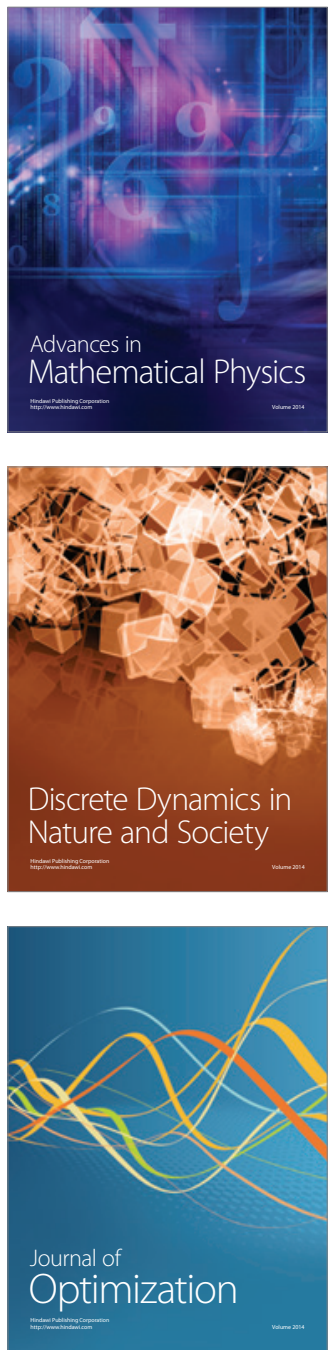